TOKYO J. MATH.

VOL. 28, No. 1, 2005

\title{
The Universality Theorem for Hecke $L$-Functions in the $(m, t)$ Aspect
}

\author{
Hidehiko MISHOU
}

(Communicated by K. Oka)

\section{Introduction}

In the paper [6], we proved certain universality theorem for Hecke $L$-functions over imaginary quadratic fields. The purpose of this paper is to extend the result for general algebraic fields.

The study of universality properties for zeta functions began with S. M. Voronin [17], in which he proved the universality property for the Riemann zeta function $\zeta(s)$. After Voronin's work, many mathematicians investigated on the universality property for other zeta functions. S. M. Gonek [5] and B. Bagchi [1] proved universality theorems for Dirichlet $L$-functions and Hurwitz zeta functions in different ways. A. Reich [16] proved universality property for Dedekind zeta functions. Recently A. Laurinčikas and K. Matsumoto [9] proved it for automorphic $L$-functions attatched with Hecke eigen cusp forms. The author proved in [11] universality theorem for Hecke $L$-functions associated with Grössencharacters. The result is the following.

THEOREM 1. Let $K$ be a finite extention of $\mathbf{Q}, n=[K: \mathbf{Q}]$, and $\chi \lambda^{m}$ be a Grössencharacter modulo $\tilde{\mathfrak{q}}$. We set

$$
\sigma_{K}= \begin{cases}\frac{1}{2} & \text { if } K=\mathbf{Q} \\ 1-\frac{1}{n} & \text { otherwise }\end{cases}
$$

Let $C$ be a simply connected compact set in the strip $\sigma_{K}<\Re s<1$ and $f(s)$ be a continuous function on $C$ such that $f(s)$ has no zero on $C$ and is analytic in the interior of $C$. Then for any $\varepsilon>0$ we have

$$
\liminf _{T \rightarrow \infty} \frac{1}{T} \mu\left\{t \in[0, T]\left|\max _{s \in C}\right| L\left(s+i t, \chi \lambda^{m}\right)-f(s) \mid<\varepsilon\right\}>0
$$

where $\mu$ is the Lebesgue measure on $\mathbf{R}$.

Received November 20, 2003; revised September 16, 2004 2000 Mathematics Subject Classification. Primary 11M41. 
Later we explain the definition of the character $\chi \lambda^{m}$ and the function $L\left(s, \chi \lambda^{m}\right)$. Roughtly speaking, this result asserts that any analytic function which satisfies the above conditions can be uniformly approximated on $C$ by vertical translation of $L\left(s, \chi \lambda^{m}\right)$ and the set of real numbers $t$ which give such approximation has a positive lower density.

The above results are concerned with the behavior of zeta functions with respect to vertical translation, that is, $t$-aspect. On the other hand there are some results on the behavior of zeta functions with respect to other parameters. Gonek [5] and Bagchi [1] independently proved that, varying Dirichlet characters as a parameter, the similar universality property holds for Dirichlet $L$-functions. Namely, for given compact set $C$ in the critical strip and an analytic function $f(s)$ on $C$, if we choose sufficiently large integer $q$, there exists a Dirichlet character $\chi$ modulo $q$ such that the associated $L$-function uniformly approximates $f(s)$ on $C$. H. Nagoshi give the similar universality property, in [13] for the parameter of holomorphic Hecke eigen cusp forms, and in [14] for the parameter of Maass cusp forms. On the other hand, Petridis-Sarnak [15] study the mean value estimate for automorphic $L$-functions twisted by Grössencharacters.

S. Koyama was inspired by these results, and has conjectured certain universality property for Hecke $L$-functions. To state it, we recall some notion about Grössencharacters. Let $K$ be a finite extention over $\mathbf{Q}$ with degree $n$, and $\mathfrak{q}$ be an integral ideal of $K$. We denote by $\mathbf{I}_{K}$ the set of fractional ideals of $K$ which is coprime to $\mathfrak{q}$. If a function $\lambda: \mathbf{I}_{K} \rightarrow \mathbf{C}^{*}=\mathbf{C}-\{0\}$ satisfies the following conditions we call it a Grösseccharacter modulo $\tilde{\mathfrak{q}}$.

(i) $\lambda(\mathfrak{a} \mathfrak{b})=\lambda(\mathfrak{a}) \lambda(\mathfrak{b}) \quad\left(\mathfrak{a}, \mathfrak{b} \in \mathbf{I}_{K}\right)$.

(ii) For $\alpha \in K, \alpha \equiv 1(\bmod \tilde{\mathfrak{q}})$ we have

$$
\lambda((\alpha))=\prod_{p=1}^{r_{1}+r_{2}}\left|\alpha^{(p)}\right|^{i v_{p}} \prod_{q=r_{1}+1}^{r_{1}+r_{2}}\left(\frac{\alpha^{(q)}}{\left|\alpha^{(q)}\right|}\right)^{u_{q}}
$$

where $r_{1}$ is the number of real embeddings of $K, 2 r_{2}$ is the number of complex embeddings of $K,\left(\alpha^{(1)}, \cdots, \alpha^{\left(r_{1}\right)}, \alpha^{\left(r_{1}+1\right)}, \cdots, \alpha^{\left(r_{1}+r_{2}\right)}, \overline{\alpha^{\left(r_{1}+1\right)}}, \cdots, \overline{\alpha^{\left(r_{1}+r_{2}\right)}}\right) \in \mathbf{R}^{r_{1}} \times \mathbf{C}^{2 r_{2}}$ denotes the set of conjugates of $\alpha, v_{p} \in \mathbf{R}$ s.t. $\sum_{p} v_{p}=0$ and $u_{q} \in \mathbf{Z}$.

It is known that any Grössencharacter $\lambda(\bmod \tilde{\mathfrak{q}})$ can be uniquely represented

$$
\lambda=\chi \lambda^{m}=\chi \lambda_{1}^{m_{1}} \cdots \lambda_{n-1}^{m_{n-1}}
$$

where $\chi$ is a narrow class group character modulo $\tilde{\mathfrak{q}}, \lambda_{1}, \cdots, \lambda_{n-1}$ is a basis of a torsion-free group generated by Grössencharacter modulo $\tilde{\mathfrak{q}}$ and $m=\left(m_{1}, \cdots, m_{n-1}\right) \in \mathbf{Z}^{n-1}$. For $\Re s>1$ we define Hecke $L$-function associated with $\chi \lambda^{m}$ by the Dirichlet series

$$
L\left(s, \chi \lambda^{m}\right)=\sum_{\mathfrak{a}} \frac{\chi \lambda^{m}(\mathfrak{a})}{N \mathfrak{a}^{s}}
$$

where $\mathfrak{a}$ runs through all integral ideals in $K$ without 0 and $N \mathfrak{a}$ is a norm of $\mathfrak{a}$. Now we state Koyama's conjecture. 
Conjecture. Let $K$ be a finite extention of $\mathbf{Q}$ such that $n=[K: \mathbf{Q}] \geq 2, \chi$ be a narrow class group character modulo $\tilde{\mathfrak{q}}$ and $\lambda_{1}, \cdots, \lambda_{n-1}$ be a basis of Grössencharacters modulo $\tilde{\mathfrak{q}}$. Let $C$ be a simply connected compact set in the strip $\sigma_{K}<\Re$ is $<1$ and $f(s)$ be a continuous function on $C$ which has non-zero on $C$ and is analytic in the interior of $C$. Then for any $\varepsilon>0$, we will have

$$
\liminf _{T \rightarrow \infty} \frac{1}{T^{n-1}} \sharp\left\{m \in \mathbf{Z}^{n-1}|| m\left|\leq T, \max _{s \in C}\right| L\left(s, \chi \lambda^{m}\right)-f(s) \mid<\varepsilon\right\}>0
$$

where $|m|=\max _{1 \leq i \leq n-1}\left|m_{i}\right|$.

Now we consider what is essential to obtain universality properties. In the case of $t$ aspect, there are two important properties on the behavior of $n^{-i t}(t \in \mathbf{R})$. One is a squaremean value estimate for Dirichlet polynomial, such as the Montgomery-Vaughn estimate

$$
\int_{0}^{T}\left|\sum_{n \leq x} \frac{a_{n}}{n^{i t}}\right|^{2} d t=T \sum_{n \leq x}\left|a_{n}\right|^{2}+O\left(\sum_{n \leq x} n\left|a_{n}\right|^{2}\right)
$$

for any $a_{n} \in \mathbf{C}$. The other is uniformly distribution of the set

$$
\left\{\left(p_{1}^{i t}, \cdots, p_{r}^{i t}\right) \in\left(S^{1}\right)^{r} \mid t \in \mathbf{R}\right\}
$$

where $p_{1}, \cdots, p_{r}$ are arbitrary prime numbers relatively prime. In the case of Dirichlet character aspect, $\chi(n)$ plays an important role instead of $n^{-i t}$ and similar properties are easily obtained by using orthogonality of Dirichlet characters. In the case of automorphic forms, $n$-th Fourier coefficient takes place of $n^{-i t}$. The mean value estimate can be obtained by the well-known Petterson's formula for Fourier coefficients and the uniformly distribution of the set of Fourier coefficients can be induced from a trace formula.

Concerning Grössencharacters, while the result on uniformly distribution has been proved in [12], the necessary large sieve type inequality

$$
\frac{1}{T^{n-1}} \sum_{|m| \leq T}\left|\sum_{N \mathfrak{a} \leq x} a(\mathfrak{a}) \chi \lambda^{m}(\mathfrak{a})\right|^{2}=\sum_{N \mathfrak{a} \leq x}|a(\mathfrak{a})|^{2}+O\left(\sum_{N \mathfrak{a} \leq x} n|a(\mathfrak{a})|^{2}\right)
$$

that is conjectured by Duke [4], is unsolved unfortunately.

In this paper, we apply the Montgomery-Vaughn estimate instead of (3) and obtain universality property with respect to $m$ and $t$ aspects simultaneously. The main result is the following.

THEOREM 2. We assume the same notation as in Conjecture. Then for any $\varepsilon>0$ we have

$$
\liminf _{T \rightarrow \infty} \frac{1}{T^{n}} \mu^{\prime}\left\{(m, t) \in[0, T]^{n}\left|\max _{s \in C}\right| L\left(s+i t, \chi \lambda^{m}\right)-f(s) \mid<\varepsilon\right\}>0
$$

where $\mu^{\prime}$ is the product measure on $\mathbf{Z}^{n-1} \times \mathbf{R}$. 
We may say that this theorem supports Koyama's conjecture when we take an average it on $t$. On the other hand, since this theorem has the same form as Theorem 1 if we average it on $m$, Theorem 2 seems easily reduced from Theorem 1. However the estimate (1) in Theorem 1 depends on $m$ unexplicitly, we can not obtain Theorem 2 from Theorem 1 directly. Conversely, from Theorem 2, the estimate (1) seems to hold uniformly for $m$.

REMARK. H. Bohr investigated the value distribution of $\zeta(s)$, in the halfplane $\mathfrak{R} s>1$ in [2] and in the strip $\frac{1}{2}<\Re s<1$ in [3] respectively. He showed that for any $\frac{1}{2}<\sigma<1$ fix, the set of $\zeta(\sigma+i t)(t \in \mathbf{R})$ is dense in $\mathbf{C}$. The universality property can be regarded an extention of Bohr's result in functional space. We consider the value distribution of Hecke $L$-functions in the $m$-aspect. For $\sigma_{K}<\sigma<1$ we need the Duke's inequality but for $\sigma>1$ we only need the result on uniformly distribution and obtain the similar result in [12]. We consider this result assures that Koyama's conjecture is true.

The author would like to express his sincere gratitude to Professor Shinya Koyama for his advice and encouragement. He would also like to express his sincere thanks to the referee for his helpful comment.

\section{Outline of proof}

We assume next lemmas.

LEMMA 1. For $z>0$ we define

$$
L_{z}\left(s, \chi \lambda^{m}\right)=\prod_{p \leq z} \prod_{\mathfrak{p} \mid p}\left(1-\frac{\chi \lambda^{m}(\mathfrak{p})}{N \mathfrak{p}^{s}}\right)^{-1}
$$

where $p$ denotes a prime number and $\mathfrak{p}$ denotes a prime ideal in $K$. For any $\varepsilon>0$ and $0<\delta^{\prime}<1$ there exists $z_{0}>0$ such that if we set

$$
A_{T, z}=\left\{(m, t) \in[0, T]^{n}\left|\max _{s \in C}\right| \log L\left(s+i t, \chi \lambda^{m}\right)-\log L_{z}\left(s+i t, \chi \lambda^{m}\right) \mid<\varepsilon\right\}
$$

where the branch of $\log L\left(s+i t, \chi \lambda^{m}\right)$ is taken by the Taylar expansion

$$
\sum_{\mathfrak{p}} \sum_{k=1}^{\infty} \frac{\chi \lambda^{m}\left(\mathfrak{p}^{k}\right)}{k N \mathfrak{p}^{k s}}
$$

on the line $\Re s=2$ and is extended analytically along the interval $[2+i \Im s+i t, s+i t]$, then for any $z>z_{0}$ we have

$$
\liminf _{T \rightarrow \infty} \frac{\mu^{\prime}\left(A_{T, z}\right)}{T^{n}}>\delta^{\prime}
$$


LEMMA 2. Let $g(s)$ be a continuous function on $C$ which is analytic in the interior of C. For any $\varepsilon>0$ there exist $0<\delta<1$ and $z_{1}>0$ such that if we set

$$
B_{T, z}=\left\{(m, t) \in[0, T]^{n}\left|\max _{s \in C}\right| \log L_{z}\left(s+i t, \chi \lambda^{m}\right)-g(s) \mid<\varepsilon\right\}
$$

then for any $z>z_{1}$ we have

$$
\liminf _{T \rightarrow \infty} \frac{\mu^{\prime}\left(B_{T, z}\right)}{T^{n}}>\delta .
$$

Let $f(s)$ be a function which satisfies conditions in Theorem 2 and $g(s)=\log f(s)$. For any $\varepsilon>0$ we take $z_{1}>0$ and $0<\delta<1$ in Lemma 2. For $\varepsilon$ and $\delta^{\prime}=1-\frac{\delta}{2}$ we take $z_{0}>0$ in Lemma 1. If we choose $z>\max \left(z_{0}, z_{1}\right)$, then we have

$$
\begin{aligned}
\max _{s \in C} \mid & \log L\left(s+i t, \chi \lambda^{m}\right)-g(s) \mid \\
\leq & \max _{s \in C}\left|\log L\left(s+i t, \chi \lambda^{m}\right)-\log L_{z}\left(s+i t, \chi \lambda^{m}\right)\right| \\
& \quad+\max _{s \in C}\left|\log L_{z}\left(s+i t, \chi \lambda^{m}\right)-g(s)\right|<2 \varepsilon
\end{aligned}
$$

for any $(m, t) \in A_{T, z} \cap B_{T, z}$, and see that $A_{T, z} \cap B_{T, z}$ has the positive lower density

$$
\liminf _{T \rightarrow \infty} \frac{1}{T^{n}} \mu^{\prime}\left(A_{T, z} \cap B_{T, z}\right)>\delta-\frac{\delta}{2}=\frac{\delta}{2}>0 .
$$

Therefore we obtain Theorem 2 from Lemma 1 and Lemma 2.

\section{The proof of Lemma 1}

To prove Lemma 1, we need to calculate a mean square estimate for $L\left(s, \chi \lambda^{m}\right)$. First we obtain an approximate functional equation for $L\left(s, \chi \lambda^{m}\right)$. If a character $\chi$ is primitive, then $L\left(s, \chi \lambda^{m}\right)$ satisfies the following functional equation

$$
L\left(s, \chi \lambda^{m}\right)=H\left(s, \chi \lambda^{m}\right) L\left(1-s, \bar{\chi} \lambda^{-m}\right)
$$

where

$$
H\left(s, \chi \lambda^{m}\right)=W\left(\chi \lambda^{m}\right) A(\mathfrak{q})^{1-2 s} \prod_{p=1}^{r_{1}} \frac{\Gamma\left(\frac{1-s+a_{p}-i b_{p}}{2}\right)}{\Gamma\left(\frac{s+a_{p}+i b_{p}}{2}\right)} \prod_{q=r_{1}+1}^{r_{1}+r_{2}} \frac{\Gamma\left(1-s+\frac{a_{q}}{2}-i b_{q}\right)}{\Gamma\left(s+\frac{a_{q}}{2}+i b_{q}\right)},
$$

the numbers $a_{1}, \cdots, a_{r_{1}} \in\{0,1\}, a_{r_{1}+1}, \cdots, a_{r_{1}+r_{2}} \in \mathbf{Z}, b_{1}, \cdots, b_{r_{1}+r_{2}} \in \mathbf{R}$ depend on $m$ and $\left|W\left(\chi \lambda^{m}\right)\right|=1$. We could derive from it the following appoximate functional equation.

Lemma 3. Let $x, y>0,0<\beta<\alpha<2,0<\gamma<2$. We denote $n$-th Dirichlet coefficient of $L\left(s, \chi \lambda^{m}\right)$ by $a_{m}(n)$ i.e. $L\left(s, \chi \lambda^{m}\right)=\sum a_{m}(n) n^{-s}$. If a character $\chi$ is primitive, then for $\beta<\Re$ is $<\alpha$ it holds that

$$
L\left(s, \chi \lambda^{m}\right)=J_{1}+J_{2}+J_{3}+J_{4}-J_{5}-J_{6}
$$


where

$$
\begin{aligned}
& J_{1}=\sum_{n \leq x} \frac{a_{m}(n)}{n^{s}}, \quad J_{2}=H\left(s, \chi \lambda^{m}\right) \sum_{n \leq y} \frac{\overline{a_{m}(n)}}{n^{1-s}} \\
& J_{3}=\sum_{n>x} \frac{a_{m}(n)}{n^{s}} e^{-\left(\frac{n}{x}\right)^{2}}, \quad J_{4}=\frac{1}{2 \pi i} \int_{(-\gamma)} x^{w} \frac{\Gamma\left(1+\frac{w}{2}\right)}{w} \sum_{n \leq x} \frac{a_{m}(n)}{n^{s+w}} d w \\
& J_{5}=\frac{1}{2 \pi i} \int_{(\beta)} H\left(s+w, \chi \lambda^{m}\right) x^{w} \frac{\Gamma\left(1+\frac{w}{2}\right)}{w} \sum_{n \leq y} \frac{\overline{a_{m}(n)}}{n^{1-s-w}} d w \\
& J_{6}=\frac{1}{2 \pi i} \int_{(-\alpha)} H\left(s+w, \chi \lambda^{m}\right) x^{w} \frac{\Gamma\left(1+\frac{w}{2}\right)}{w} \sum_{n>y} \frac{\frac{a_{m}(n)}{n^{1-s-w}}}{w} d w .
\end{aligned}
$$

ProOF. For $X>0$ we have

$$
e^{-X^{2}}=\frac{1}{2 \pi i} \int_{(1)} X^{-w} \frac{\Gamma\left(1+\frac{w}{2}\right)}{w} d w .
$$

We take $X=\frac{n}{x}$ in (4) for each $n$ and combine them, then

$$
\begin{aligned}
\sum_{n=1}^{\infty} \frac{a_{m}(n)}{n^{s}} e^{-\left(\frac{n}{x}\right)^{2}} & =\sum_{n=1}^{\infty} \frac{a_{m}(n)}{n^{s}} \cdot \frac{1}{2 \pi i} \int_{(1)}\left(\frac{x}{n}\right)^{w} \frac{\Gamma\left(1+\frac{w}{2}\right)}{w} d w \\
& =\frac{1}{2 \pi i} \int_{(1)} L\left(s+w, \chi \lambda^{m}\right) x^{w} \frac{\Gamma\left(1+\frac{w}{2}\right)}{w} d w
\end{aligned}
$$

where interchange of integral and series is permitted because of absolute convergence of the series $\sum a_{m}(n) n^{-s}$ in the halfplane $\Re s>1$. We move contour from $\Re w=1$ to $\Re w=-\alpha$. Since the integrand has a pole at $w=0$ with a residue $L\left(s, \chi \lambda^{m}\right)$, we have

(5) $\quad L\left(s, \chi \lambda^{m}\right)$

$$
=\sum_{n \leq x} \frac{a_{m}(n)}{n^{s}} e^{-\left(\frac{n}{x}\right)^{2}}+J_{3}-\frac{1}{2 \pi i} \int_{(-\alpha)} L\left(s+w, \chi \lambda^{m}\right) x^{w} \frac{\Gamma\left(1+\frac{w}{2}\right)}{w} d w .
$$

We apply the functional equation to the third term. Since $\Re(1-s-w)>1-\alpha+\alpha=1$ we have

$$
\begin{aligned}
& \frac{1}{2 \pi i} \int_{(-\alpha)} L\left(s+w, \chi \lambda^{m}\right) x^{w} \frac{\Gamma\left(1+\frac{w}{2}\right)}{w} d w \\
& \quad=\frac{1}{2 \pi i} \int_{(-\alpha)} H\left(s+w, \chi \lambda^{m}\right) L\left(1-s-w, \bar{\chi} \lambda^{-m}\right) x^{w} \frac{\Gamma\left(1+\frac{w}{2}\right)}{w} d w \\
& \quad=J_{6}+\frac{1}{2 \pi i} \int_{(-\alpha)} H\left(s+w, \chi \lambda^{m}\right) x \frac{\Gamma\left(1+\frac{w}{2}\right)}{w} \sum_{n \leq y} \frac{\overline{a_{m}(n)}}{n^{1-s-w}} d w
\end{aligned}
$$


We move a contour from $\Re w=-\alpha$ to $\Re w=\beta$. Since the integrand has a pole at $w=0$ with residue $H\left(s, \chi \lambda^{m}\right) \sum_{n \leq y} \overline{a_{m}(n)} n^{s-1}$, the third term in (5) is equal to $J_{2}-J_{5}-J_{6}$. We return to the equality (4). If we move a contour from $\Re w=1$ to $\Re w=-\gamma$ then we have

$$
e^{-X^{2}}=1+\frac{1}{2 \pi i} \int_{(-\gamma)} X^{-w} \frac{\Gamma\left(1+\frac{w}{2}\right)}{w} d w
$$

We put $X=\frac{n}{x}$ for each $n \leq x$ and combine them, then

$$
\sum_{n \leq x} \frac{a_{m}(n)}{n^{s}} e^{-\left(\frac{n}{x}\right)^{2}}=J_{1}+J_{4}
$$

Therefore we have the approximate functional equation.

Now we prove Lemma 1. First we assume that $\chi$ is primitive. Instead of the inequality in the statement, it is enough to consider the inequality

$$
\max _{s \in C}\left|L_{z}^{-1}\left(s+i t, \chi \lambda^{m}\right) \cdot L\left(s+i t, \chi \lambda^{m}\right)-1\right|<\varepsilon
$$

We take a compact set $C_{1}$ in the strip $\sigma_{K}<\Re s<1$ such that $C \subset C_{1}$. From elementaly functional theory (refer to Appendix of [7], for example), if the inequality

$$
\iint_{C_{1}}\left|L_{z}^{-1}\left(s+i t, \chi \lambda^{m}\right) \cdot L\left(s+i t, \chi \lambda^{m}\right)-1\right|^{2} d \sigma d \tau<\varepsilon^{2} \quad(s=\sigma+i \tau)
$$

holds, then we have

$$
\max _{s \in C}\left|L_{z}^{-1}\left(s+i t, \chi \lambda^{m}\right) \cdot L\left(s+i t, \chi \lambda^{m}\right)-1\right|<c\left(C, C_{1}\right) \varepsilon
$$

Therefore we will prove the positive density of the set

$$
B_{T, z}^{\prime}=\left\{(m, t) \in[0, T]^{n}\left|\iint_{C_{1}}\right| L_{z}^{-1}\left(s+i t, \chi \lambda^{m}\right) \cdot L\left(s+i t, \chi \lambda^{m}\right)-\left.1\right|^{2} d \sigma d \tau<\varepsilon^{2}\right\}
$$

We calculate the second moment

$$
\sum_{\substack{T<m_{i} \leq 2 T \\(1 \leq i \leq n-1)}} \int_{T}^{2 T} \iint_{C_{1}}\left|L_{z}^{-1}\left(s+i t, \chi \lambda^{m}\right) \cdot L\left(s+i t, \chi \lambda^{m}\right)-1\right|^{2} d \sigma d \tau d t
$$


Let $s=\sigma+i \tau \in C_{1}$ fix. By using Lemma 3 we have

(7)

$$
\begin{gathered}
\sum_{\substack{T<m_{i} \leq 2 T \\
(1 \leq i \leq n-1)}} \int_{T}^{2 T}\left|L_{z}^{-1}\left(s+i t, \chi \lambda^{m}\right) \cdot L\left(s+i t, \chi \lambda^{m}\right)-1\right|^{2} d t \\
\ll \sum_{T<m_{i} \leq 2 T} \int_{T}^{2 T}\left|L_{z}^{-1}\left(s+i t, \chi \lambda^{m}\right) \cdot J_{1}-1\right|^{2} d t
\end{gathered}
$$

$$
\begin{aligned}
& +\sum_{j=2}^{6} \sum_{T<m_{i} \leq 2 T} \int_{T}^{2 T}\left|L_{z}^{-1}\left(s+i t, \chi \lambda^{m}\right) \cdot J_{j}\right|^{2} d t \\
= & I_{1}+\sum_{j=2}^{6} I_{j} .
\end{aligned}
$$

First we consider the integral $I_{1}$. It holds that

$$
\prod_{p \leq z \mathfrak{p} \mid p}\left(1-\frac{\chi \lambda^{m}(\mathfrak{p})}{N \mathfrak{p}^{s}}\right)^{-1} \cdot \sum_{N \mathfrak{a} \leq x} \frac{\chi \lambda^{m}(\mathfrak{a})}{N \mathfrak{a}^{s}}=1+\sum_{z<n \leq x z^{\prime}} \frac{b_{n}(m)}{n^{s}}
$$

where $z^{\prime}$ only depends on $z$ and $b_{m}(n) \ll n^{\varepsilon}$ uniformly in $m$. Taking $x=T^{\frac{n}{2}}$ and applying the Montgomery-Vaughn estimate (2), we have

$$
\begin{aligned}
I_{1} & =\sum_{T<m_{i} \leq 2 T} \int_{T}^{2 T}\left|\sum_{z<n \leq x z^{\prime}} \frac{b_{m}(n)}{n^{s+i t}}\right|^{2} d t \\
& \ll T^{n-1}\left\{T \sum_{z<n \leq x z^{\prime}} \frac{1}{n^{2 \sigma-\varepsilon}}+\sum_{z<n \leq x z^{\prime}} \frac{1}{n^{2 \sigma-\varepsilon-1}}\right\} \\
& \ll T^{n}\left(z^{1-2 \sigma+\varepsilon}+z^{\prime \prime} T^{-1+n-n \sigma+\varepsilon}\right),
\end{aligned}
$$

where $z^{\prime \prime}$ depends on $z$ and $\sigma$. Next we consider the integrals $I_{2}, I_{4}$ and $I_{5}$. By using Stirling formul

$$
\log \Gamma(s)=\left(s-\frac{1}{2}\right) \log s-s+\log \sqrt{2 \pi}+O\left(|s|^{-1}\right),
$$

the following estimates

$$
\begin{gathered}
H\left(s+i t, \chi \lambda^{m}\right) \ll T^{n\left(\frac{1}{2}-\sigma\right)}, \\
\int_{(-\gamma)}\left|\frac{\Gamma\left(1+\frac{w}{2}\right)}{w}\right| d w \ll 1, \\
\int_{(\beta)}\left|H\left(s+w, \chi \lambda^{m}\right) \frac{\Gamma\left(1+\frac{w}{2}\right)}{w}\right| d w \ll T^{\frac{n}{2}(1-2 \sigma-2 \beta)}
\end{gathered}
$$


hold for $T<m_{i} \leq 2 T(1 \leq i \leq n-1)$ and $T<t<2 T$ uniformly. Therefore, taking $y=T^{\frac{n}{2}}$, we have

$$
I_{2}=\sum_{|m| \leq T} \int_{T}^{2 T}\left|H\left(s+i t, \chi \lambda^{m}\right) \sum_{n \leq y} \frac{\overline{a_{m}(n)}}{n^{1-s}}\right|^{2} \cdot\left|L_{z}^{-1}\left(s+i t, \chi \lambda^{m}\right)\right|^{2} d t
$$

$$
\begin{aligned}
& \ll c(z) T^{n-1} T^{n(1-2 \sigma)}\left\{T \sum_{n \leq y} \frac{1}{n^{2-2 \sigma-\varepsilon}}+\sum_{n \leq y} \frac{1}{n^{1-2 \sigma-\varepsilon}}\right\} \\
& \ll c(z) T^{n} \cdot T^{n-n \sigma-1+\varepsilon} .
\end{aligned}
$$

Similarly we also have

$$
I_{4}, \quad I_{5} \ll_{z} T^{n} \cdot T^{n-n \sigma-1+\varepsilon}
$$

By Cauchy-Schwarz inequality

$$
\begin{aligned}
\left|\sum_{n>x} a_{n}\right|^{2} & =\left|\sum_{j=1}^{\infty} \sum_{j x<n \leq(j+1) x} a_{n}\right|^{2}=\left|\sum_{j=1}^{\infty} j^{-\frac{1+\varepsilon}{2}} j^{\frac{1+\varepsilon}{2}} \sum_{j x<n \leq(j+1) x} a_{n}\right|^{2} \\
& \ll\left(\sum_{j} j^{-1-\varepsilon}\right)\left(\sum_{j} j^{1+\varepsilon}\left|\sum_{j x<n \leq(j+1) x} a_{n}\right|^{2}\right) \\
& \ll \varepsilon\left(\sum_{j=1}^{\infty} j^{1+\varepsilon}\left|\sum_{j x<n \leq(j+1) x} a_{n}\right|^{2}\right) .
\end{aligned}
$$

We apply this inequality to the integral $I_{3}$, then

$$
\begin{aligned}
I_{3} & =\sum_{|m| \leq T} \int_{T}^{2 T}\left|\sum_{n>x} \frac{a_{m}(n)}{n^{s+i t}} e^{-\left(\frac{n}{x}\right)^{2}}\right|^{2} \cdot\left|L_{z}^{-1}\left(s+i t, \chi \lambda^{m}\right)\right|^{2} d t \\
& \ll_{z} \sum_{j=1}^{\infty} j^{1+\varepsilon} \sum_{|m| \leq T} \int_{T}^{2 T}\left|\sum_{j x<n \leq(j+1) x} \frac{a_{m}(n)}{n^{s+i t}} e^{-\left(\frac{n}{x}\right)^{2}}\right|^{2} d t \\
& \ll_{z} T^{n-1} \sum_{j=1}^{\infty} j^{1+\varepsilon} e^{-j^{2}} \int_{T}^{2 T}\left|\sum_{j x<n \leq(J+1) x} \frac{a_{m}(n)}{n^{s+i t}}\right|^{2} d t \\
& \ll_{z} T^{n-1} \sum_{j=1}^{\infty} j^{1+\varepsilon} e^{-j^{2}}\left\{T\left(j T^{\frac{n}{2}}\right)^{1-2 \sigma+\varepsilon}+\left(j T^{\frac{n}{2}}\right)^{2-2 \sigma+\varepsilon}\right\} \\
& \ll_{z} T^{n} \cdot T^{n-n \sigma-1+\varepsilon} .
\end{aligned}
$$

Similarly we obtain

$$
I_{6} \ll_{z} T^{n} \cdot T^{n-n \sigma-1+\varepsilon}
$$


From (7), (8), (9), (10), (11) and (12), we have

$$
\begin{aligned}
& \sum_{T<m_{i} \leq 2 T} \int_{T}^{2 T} \iint_{C_{1}}\left|L_{z}^{-1}\left(s+i t, \chi \lambda^{m}\right) \cdot L\left(s+i t, \chi \lambda^{m}\right)-1\right|^{2} d \sigma d \tau d t \\
& \quad \ll C_{1} T^{n}\left(z^{1-2 \sigma_{1}+\varepsilon}+c(z) T^{n-n \sigma_{1}-1+\varepsilon}\right)
\end{aligned}
$$

where $\sigma_{1}=\max _{s \in C_{1}} \sigma$. Since $\sigma_{1}>1-\frac{1}{n}$, if we take $z_{0}>0$ sufficiently large, then for any $z>z_{0}$ and $T>T(z)$ we have

$$
\begin{aligned}
& \frac{1}{T^{n}} \sum_{T<m_{i} \leq 2 T} \int_{T}^{2 T} \iint_{C_{1}}\left|L_{z}^{-1}\left(s+i t, \chi \lambda^{m}\right) \cdot L\left(s+i t, \chi \lambda^{m}\right)-1\right|^{2} d \sigma d \tau d t \\
& \quad<\varepsilon^{3}\left(1-\delta^{\prime}\right) .
\end{aligned}
$$

We assume that the measure of the set $B_{T, z}^{\prime}$ is smaller than $\delta^{\prime} T^{n}$. It implis that

$$
\begin{aligned}
& \frac{1}{T^{n}} \mu^{\prime}\left\{(m, t) \in[0, T]^{n} \mid \iint_{C_{1}}\right. \\
& \left.\quad \times\left|L_{z}^{-1}\left(s+i t, \chi \lambda^{m}\right) \cdot L\left(s+i t, \chi \lambda^{m}\right)-1\right|^{2} d \sigma d \tau \geq \varepsilon^{2}\right\}>1-\delta^{\prime}
\end{aligned}
$$

It contradicts (12) clearly. Hence we obtain Lemma 1 when $\chi$ is primitive. In general case, let $\chi_{1}\left(\bmod \tilde{\mathfrak{q}}_{1}\right)$ be a primitive character induced by $\chi$, then it holds that

$$
L\left(s, \chi \lambda^{m}\right)=\prod_{\mathfrak{p} \mid \mathfrak{q}}\left(1-\frac{\chi_{1} \lambda^{m}(\mathfrak{p})}{N \mathfrak{p}^{s}}\right) L\left(s, \chi_{1} \lambda^{m}\right)
$$

For sufficiently large $z$, we have the similar formula between $L_{z}\left(s, \chi \lambda^{m}\right)$ and $L_{z}\left(s, \chi_{1} \lambda^{m}\right)$. By these formula, we have

$$
\log L\left(s, \chi \lambda^{m}\right)-\log L_{z}\left(s, \chi \lambda^{m}\right)=\log L\left(s, \chi_{1} \lambda^{m}\right)-\log L_{z}\left(s, \chi_{1} \lambda^{m}\right) .
$$

Therefore we complete the proof of Lemma 1.

\section{The proof of Lemma 2}

The next result plays an essencial role in the study of value distribution of zeta functions with respect to $t$-aspect.

LEMMA 4. Let $p_{1}, \cdots, p_{r}$ be prime numbers relatively prime. Then a $r$-dimention curve

$$
\gamma(t)=\left(t \log p_{1}, \cdots, t \log p_{r}\right) \quad(t \in \mathbf{R})
$$

is uniformly distributed $(\bmod 1)$. More precisely, we have the following two properties. 
(i) For every Jordan measurable set A of the unit cube in $\mathbf{R}^{r}$, it holds that

$$
\lim _{T \rightarrow \infty} \frac{1}{T} \mu\{t \in[0, T] \mid \gamma(t) \in A \quad(\bmod 1)\}=m(A)
$$

where $m(A)$ is the volume of $A$.

(ii) Let $D$ be a closed and Jordan measurable subregion of the unit cube in $\mathbf{R}^{r}$ and $\Omega$ be a set of complex valued functions on $D$. If $\Omega$ is uniformly bounded and equicontinuous, then the following relation holds uniformly with respect to $f \in \Omega$

$$
\lim _{T \rightarrow \infty} \frac{1}{T} \int^{\prime} f(\{\gamma(t)\}) d t=\int_{D} f(x) d x_{1} \cdots d x_{r}
$$

where $\int^{\prime}$ denotes the integral over $t \in(0, T)$ for which $\gamma(t) \in D(\bmod 1)$.

This lemma is easily obtained by the fact that $\log p_{i}$ are linearly independent over $\mathbf{Q}$ and the Kronecker-Weyl theorem. We refer to Appendix of [7] in detail.

For a prime number $p$, we denote by $x_{p}$ the number of different prime factors of $p$ in $K$ and $\mathfrak{p}_{p, 1}, \cdots, \mathfrak{p}_{p, x_{p}}$ such prime factors. We define the set

$$
\mathbf{P}=\left\{\mathfrak{p}_{p, i} \mid(\mathfrak{q}, p)=1,1 \leq i \leq x_{p}-1\right\} \cup\left\{\mathfrak{p}_{p, i} \mid(\mathfrak{q}, p) \neq 1,\left(\mathfrak{q}, \mathfrak{p}_{p, i}\right)=1\right\} .
$$

It means that for any $\mathfrak{p}_{1}, \cdots, \mathfrak{p}_{r} \in \mathbf{P}$, there is no prime number $p$ which divides any ideals generated by $\mathfrak{p}_{i}$. We quote Lemma 2 in [12].

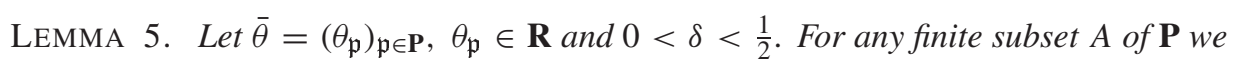
have

$$
\lim _{T \rightarrow \infty} \frac{1}{(2 T)^{n-1}} \sharp\left\{|m| \leq T \mid\left\|\theta_{\mathfrak{p}}-\frac{1}{2 \pi} \arg \lambda^{m}(\mathfrak{p})\right\|<\delta(\mathfrak{p} \in A)\right\}=(2 \delta)^{\sharp A}
$$

where $\|y\|=\min _{n \in \mathbf{Z}}|y-n|$.

This lemma is an analogue of Lemma 4 with respect to $m$-aspect. It is proved by using the uniqueness of the factorization into prime ideals and the definition of Grössencharacters.

Now we prove Lemma 2. We denote the decomposition of a prime number $p$ in $K$

$$
p=\mathfrak{p}_{p, 1}^{y_{1}} \cdots \mathfrak{p}_{p, x_{p}}^{y_{x_{p}}}, \quad N \mathfrak{p}_{p, i}=p^{z_{i}}
$$

Then we have

$$
\begin{aligned}
\log L_{z}\left(s, \chi \lambda^{m}\right) & =\sum_{p \leq z} \sum_{i=1}^{x_{p}} \log \left(1-\frac{\chi \lambda^{m}\left(\mathfrak{p}_{p, i}\right)}{p^{z_{i} s}}\right)^{-1} \\
& =\sum_{p \leq z} \sum_{i=1}^{x_{p}} \sum_{k=1}^{\infty} \frac{\chi \lambda^{m}\left(\mathfrak{p}_{p, i}^{k}\right)}{k p^{k z_{i} s}}
\end{aligned}
$$


We take sufficiently large $y>0$ and split the sum over $p \leq z$ into the ones over $p \leq y$ and $y<p \leq z$. Let $N=[2 \log y]$ and divide the sum over $k$ into three parts, $k=1,2 \leq k \leq N$ and $k>N$. For partial sums, we have the estimates

$$
\begin{gathered}
\sum_{y<p \leq z} \sum_{i=1}^{x_{p}} \sum_{k=2}^{\infty} \frac{\chi \lambda^{m}\left(\mathfrak{p}_{p, i}^{k}\right)}{k p^{k z_{i} s}} \ll_{K} \sum_{y<p} \frac{1}{p^{2 \sigma}} \ll y^{1-2 \sigma}, \\
\sum_{p \leq y} \sum_{i=1}^{x_{p}} \sum_{k>N} \frac{\chi \lambda^{m}\left(\mathfrak{p}_{p, i}^{k}\right)}{k p^{k z_{i} s}} \ll_{K} y 2^{-N \sigma} \ll y^{1-2 \sigma} .
\end{gathered}
$$

Hence

$$
\log L_{z}\left(s, \chi \lambda^{m}\right)=\sum_{y<p \leq z} \sum_{i=1}^{x_{p}} \frac{\chi \lambda^{m}\left(\mathfrak{p}_{p, i}\right)}{p^{z_{i} s}}+l(s, y, m)+O\left(y^{1-2 \sigma}\right)
$$

where

$$
l(s, y, m)=\sum_{p \leq y} \sum_{i=1}^{x_{p}} \sum_{k \leq N} \frac{\chi \lambda^{m}\left(\mathfrak{p}_{p, i}^{k}\right)}{k p^{k z_{i} s}} .
$$

Now we introduce the denseness lemma.

LEMMA 6. Let $y>0, C$ be a simply connected compact set in the critical strip $\frac{1}{2}<$ $\Re s<1$ and $h(s)$ be a continuous function on $C$ which is analytic in the interior of $C$. Then there exists $v_{0}=v_{0}(C, h, y)>0$ such that for any $v>v_{0}$ we can choose $\theta_{p} \in[0,1)(y<$ $p \leq v)$ for which

$$
\max _{s \in C}\left|\sum_{y<p \leq \nu} \sum_{i=1}^{x_{p}} \frac{\chi\left(\mathfrak{p}_{p, i}\right) e\left(z_{i} \theta_{p}\right)}{p^{z_{i} s}}-h(s)\right| \ll y^{-\frac{1}{2}}
$$

holds.

PROOF. By applying the general denseness lemma in [8], it is enough to prove a positive density of the set

$$
\left\{p \text { :prime number }|| \sum_{i=1}^{x_{p}} \chi\left(\mathfrak{p}_{p, i}\right) \mid>\eta\right\}
$$

for some $\eta>0$. It is assured by the decomposition theorem of class field theory. We refer to section 4 in [10] in detail.

For sufficiently large $y>0$ we set

$$
h(s)=g(s)-l(s, y, 0)
$$


and take $v>v_{0}(y, C, h)$ such that

$$
v^{1-2 \sigma}<\frac{1}{2} \varepsilon^{3}
$$

We choose $\theta_{p} \in[0,1)$ for $h(s)$ and $v$. Then by (14) and Lemma 6 we have

$$
\begin{aligned}
\max _{s \in C} \mid & \log L_{z}\left(s+i t, \chi \lambda^{m}\right)-g(s) \mid \\
\leq & \max _{s \in C} \mid \sum_{y<p \leq z} \sum_{i=1}^{x_{p}} \frac{\chi \lambda^{m}\left(\mathfrak{p}_{p, i}\right)}{p^{z_{i}(s+i t)}+l(s+i t, y, m)-g(s) \mid+\varepsilon} \\
\leq & \max _{s \in C}\left|\sum_{y<p \leq z} \sum_{i=1}^{x_{p}} \frac{\chi \lambda^{m}\left(\mathfrak{p}_{p, i}\right)}{p^{z_{i}(s+i t)}}-\sum_{y<p \leq z} \sum_{i=1}^{x_{p}} \frac{\chi\left(\mathfrak{p}_{p, i}\right) e\left(z_{i} \theta_{p}\right)}{p^{z_{i} s}}\right| \\
& +\max _{s \in C}|l(s+i t, y, m)-l(s, y, 0)|+\max _{s \in C}\left|\sum_{\nu<p \leq z} \sum_{i=1}^{x_{p}} \frac{\chi \lambda^{m}\left(\mathfrak{p}_{p, i}\right)}{p^{z_{i} s}}\right|+\varepsilon .
\end{aligned}
$$

Let $h(\tilde{\mathfrak{q}})$ be the ray class number modulo $\tilde{\mathfrak{q}}$. Then for any $m \in \mathbf{Z}^{n-1},\left(\lambda^{m}\right)^{h(\tilde{\mathfrak{q}})}$ is trivial on $\mathbf{Q}$. For a prime number $p$

$$
0=\left\|\frac{1}{2 \pi} \arg \lambda^{h(\tilde{\mathfrak{q}}) m}(p)\right\|=\left\|\sum_{i=1}^{x_{p}} \frac{1}{2 \pi} \arg \lambda^{h(\tilde{\mathfrak{q}}) m}\left(\mathfrak{p}_{p, i}^{y_{i}}\right)\right\|
$$

where $h(\tilde{\mathfrak{q}}) m=\left(h(\tilde{\mathfrak{q}}) m_{1}, \cdots, h(\tilde{\mathfrak{q}}) m_{n-1}\right)$. Hence if we assume

$$
\left\|\frac{1}{2 \pi} \arg \lambda^{h(\tilde{\mathfrak{q}}) m}\left(\mathfrak{p}_{p, i}\right)\right\|<\frac{\delta}{n} \quad\left(1 \leq i \leq x_{p}-1\right)
$$

for sufficiently small $\delta>0$, it follows that

$$
\left\|\frac{1}{2 \pi} \arg \lambda^{h(\tilde{\mathfrak{q}}) m}\left(\mathfrak{p}_{p, x_{p}}\right)\right\|<\delta .
$$

Therefore if we choose sufficiently small $\delta>0$ against $\varepsilon^{\prime}>0$ and define the sets

$$
\begin{aligned}
& V_{T}=\left\{t \in[0, T] \mid \begin{array}{ll}
\left\|t \frac{1}{2 \pi} \log p\right\|<\delta & (p \leq y) \\
\left\|t \frac{1}{2 \pi} \log p-\theta_{p}\right\|<\delta & (y<p \leq v)
\end{array}\right\}, \\
& U_{T}=\left\{|m| \leq T \mid\left\|\frac{1}{2 \pi} \arg \lambda^{h(\tilde{\mathfrak{q}}) m}\left(\mathfrak{p}_{p, i}\right)\right\|<\delta \quad\left(\begin{array}{c}
p \leq v \\
1 \leq i \leq x_{p}-1
\end{array}\right)\right\},
\end{aligned}
$$

then for any $(t, m) \in V_{T} \times U_{T}$ we have

$$
\left|\frac{\chi \lambda^{m}\left(\mathfrak{p}_{p, i}\right)}{p^{z_{i}(s+i t)}}-\frac{\chi\left(\mathfrak{p}_{p, i}\right)}{p^{z_{i} s}}\right|<\varepsilon^{\prime} \quad(p \leq y)
$$


and

$$
\left|\frac{\chi \lambda^{m}\left(\mathfrak{p}_{p, i}\right)}{p^{z_{i}(s+i t)}}-\frac{\chi\left(\mathfrak{p}_{p, i}\right) e\left(z_{i} \theta_{p}\right)}{p^{z_{i} s}}\right|<\varepsilon^{\prime} \quad(y<p \leq v) .
$$

Further if we take sufficiently small $\varepsilon^{\prime}$ for $\varepsilon$, then the left-hand side of (14) is

$$
<\varepsilon+\max _{s \in C}\left|\sum_{\nu<p \leq z} \sum_{i=1}^{x_{p}} \frac{\chi \lambda^{m}\left(\mathfrak{p}_{p, i}\right)}{p^{z_{i}(s+i t)}}\right|
$$

for $(t, m) \in V_{T} \times U_{T}$. According to Lemma 4(i) and Lemma 5, we have the positive density

$$
\lim _{T \rightarrow \infty} \frac{\mu^{\prime}\left(V_{T} \times U_{T}\right)}{T^{n}}=\beta>0
$$

where we note that $\beta$ does not depend on $z$.

Lastly we show that the third term of the right-hand side of (14) is small enough for almost all $(t, m) \in V_{T} \times U_{T}$. We consider the second moment

$$
\sum_{m \in U_{T}} \int_{V_{T}} \iint_{C}\left|\sum_{\nu<p \leq z} \sum_{i=1}^{x_{p}} \frac{\chi \lambda^{m}\left(\mathfrak{p}_{p, i}\right)}{p^{z_{i}(s+i t)}}\right|^{2} d \sigma d \tau d t
$$

and the set

$$
\Omega=\left\{f(u)=\sum_{\nu<p \leq z} \sum_{i=1}^{x_{p}} \frac{\chi \lambda^{m}\left(\mathfrak{p}_{p, i}\right) e\left(z_{i} u_{p}\right)}{p^{z_{i} s}} \mid m \in U_{T}, s \in C\right\}
$$

where $u=\left(u_{p}\right), u_{p} \in \mathbf{R}$. Because of $\left|\chi \lambda^{m}\left(\mathfrak{p}_{p, i}\right)\right|=1$, the set $\Omega$ is uniformly bounded and equicontinuous. Hence we apply Lemma 4(ii) and have

$$
\begin{aligned}
\int^{\prime}\left|\sum_{\nu<p \leq z} \sum_{i=1}^{x_{p}} \frac{\chi \lambda^{m}\left(\mathfrak{p}_{p, i}\right)}{p^{z_{i}(s+i t)}}\right|^{2} d t & =\mu\left(V_{T}\right) \times \int_{0}^{1} \cdots \int_{0}^{1}\left|\sum_{\nu<p \leq z} \sum_{i=1}^{x_{p}} \frac{\chi \lambda^{m}\left(\mathfrak{p}_{p, i}\right) e\left(z_{i} u_{p}\right)}{p^{z_{i} s}}\right|^{2} d u \\
& \ll \mu\left(V_{T}\right) \times \sum_{\nu<p} \frac{1}{p^{2 \sigma}} \ll \mu\left(V_{T}\right) \nu^{1-2 \sigma}
\end{aligned}
$$

for $m \in U_{T}$ uniformly. Hence we have

$$
\sum_{m \in U_{T}} \int_{V_{T}} \iint_{C}\left|\sum_{\nu<p \leq z} \sum_{i=1}^{x_{p}} \frac{\chi \lambda^{m}\left(\mathfrak{p}_{p, i}\right)}{p^{z_{i}(s+i t)}}\right|^{2} d \sigma d \tau d t<\mu^{\prime}\left(U_{T} \times V_{T}\right) \frac{\varepsilon^{3}}{2} .
$$

By the similar argument in the proof of Lemma 1, there exist subsets $U_{T}^{\prime} \subset U_{T}$ and $V_{T}^{\prime} \subset V_{T}$ which have the positive density

$$
\lim _{T \rightarrow \infty} \frac{\mu^{\prime}\left(V_{T}^{\prime} \times U_{T}^{\prime}\right)}{T^{n}}>\frac{\beta}{2}>0 .
$$


For any $(m, t) \in U_{T}^{\prime} \times V_{T}^{\prime}$ and $z>z_{1}$ we have

$$
\max _{s \in C}\left|\sum_{\nu<p \leq z} \sum_{i=1}^{x_{p}} \frac{\chi \lambda^{m}\left(\mathfrak{p}_{p, i}\right)}{p^{z_{i}(s+i t)}}\right|<\varepsilon .
$$

Therefore the proof of Lemma 2 is completed.

\section{References}

[ 1 ] B. BAGCHI, The statistical behavior and universality properties of the Rieman zeta-function and other allied Dirichlet series, Ph. D. Thesis, Calcutta, Indian Statistical Institute (1981).

[ 2 ] H. Bohr, Über das Verhalten von $\zeta(s)$ in der Halbebene $\sigma>1$, Nachr. Akad. Wiss. Göttingen II Math.-phys. K1., (1911), 409-428.

[ 3 ] H. Bohr, Zur Theorie der Riemann'schen Zeta-funktion im kritischen Streifen, Acta Math. 40 (1915), 67100.

[ 4 ] W. DuKE, Some problems in multidimentional analytic number theory, Acta. Arith. 52 (1989), 203-228

[ 5 ] S. M. GoneK, Analytic properties of zeta and L-functions, Ph. D. Thesis, University of Michigan. (1979).

[ 6] S. KoYAma and H. Mishou, Universality of Hecke $L$-functions in the Grössencharacter-aspect, Proc. Japan Acad., 78, Ser. A (2002), 63-67.

[ 7 ] A. A.Karatsuba and S. M. Voronin, The Riemann Zeta-Function, Walter de Gruyter (1992).

[ 8 ] A. LAURINČIKAS, On the Matsumoto zeta-function, Acta Arith. 84 (1998), 1-16.

[9] A. LAURINČIKAS and K. Matsumoto, The universality of zeta-functions attached to certain cusp forms, Acta Arith. 98 (2001), 345-359.

[10] H. Mishou, The universality theorem for $L$-functions associated with ideal class characters, Acta Arith. 98 (2001), 395-410.

[11] H. Mishou, The universality theorem for Hecke $L$-functions, Acta Arith. 110 (2003), 45-71.

[12] H. Mishou, The value distribution of Hecke $L$-functions in the Grössencharacter aspect, Arch. Math. 82 (2004), 301-310.

[13] H. NAGOSHI, The universality of families of automorphic $L$-functions, preprint.

[14] H. NAGOSHI, On the universality for $L$-functions attached to Maass forms, preprint.

[15] Y. PETRIDIS and P. SARNAK, Quantum unique ergordicity for $S L_{2}(\mathfrak{O}) \backslash H^{3}$ and estimates for $L$-functions, J. Evol. Equ. 1 (2001), 277-290.

[16] A. ReICH, Werteverteilung von Zetafunktionen, Arch. Math. 34 (1980), 440-451.

[17] S. M. Voronin, Theorem on the "universality" of the Riemann zeta-function, Math. USSR-Izv. 9 (1975), 475-486.

Present Address:

7-49-1 AOYAGI-CHO, CHIKUSA-KU,

NAGOYA-CITY, AICHI, 464-0852 JAPAN.

e-mail:m98018a@math.nagoya-u.ac.jp 